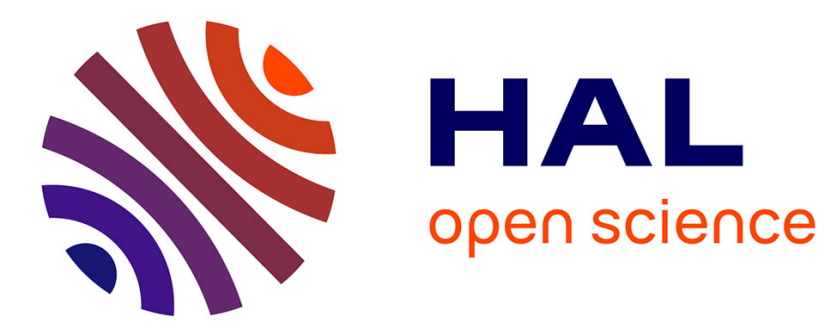

\title{
New Macrocell Downlink Energy Consumption Minimization with Cell DTx and Power Control
}

Rémi Bonnefoi, Christophe Moy, Jacques Palicot

\section{To cite this version:}

Rémi Bonnefoi, Christophe Moy, Jacques Palicot. New Macrocell Downlink Energy Consumption Minimization with Cell DTx and Power Control. IEEE International Conference on Communications (ICC 2017), May 2017, Paris, France. 10.1109/ICC.2017.7997008 . hal-01486616v3

\section{HAL Id: hal-01486616 https://hal.science/hal-01486616v3}

Submitted on 19 Oct 2018

HAL is a multi-disciplinary open access archive for the deposit and dissemination of scientific research documents, whether they are published or not. The documents may come from teaching and research institutions in France or abroad, or from public or private research centers.
L'archive ouverte pluridisciplinaire HAL, est destinée au dépôt et à la diffusion de documents scientifiques de niveau recherche, publiés ou non, émanant des établissements d'enseignement et de recherche français ou étrangers, des laboratoires publics ou privés. 


\title{
New Macrocell Downlink Energy Consumption Minimization with Cell DTx and Power Control
}

\author{
Rémi Bonnefoi, Christophe Moy and Jacques Palicot \\ CentraleSupélec/IETR, CentraleSupélec Campus de Rennes, 35510 Cesson-Sévigné, France \\ Email:\{remi.bonnefoi, christophe.moy, jacques.palicot\}@ centralesupelec.fr
}

\begin{abstract}
Power control (PC) and discontinuous transmission (DTx) can reduce the power consumption of a base station (BS). When both are used, there is, for each user, a trade-off between service time and transmit power in order to minimize the energy consumption of the base station. In this paper, we analyse this trade-off and we propose a new efficient algorithm for the computation of the optimal service time and transmit power of all users. We show that in most cases, closed-form expressions can be used. For others, we prove that the search for the optimum can be changed into a root-finding problem which can be solved efficiently with the Newton's method. Numerical results show that, compared to the use of DTx only, the proposed strategy allows to save up to $4 \%(7 \mathrm{~W})$ of the total energy consumption.
\end{abstract}

\section{INTRODUCTION}

The reduction of electricity consumption is one of the major challenges of future wireless networks. Base stations (BS) represent $40-50 \%$ of the electricity consumed by cellular networks [1]. Therefore, reducing their energy consumption will greatly decrease the operating cost for mobile operators and the carbon footprint of information and communication technologies (ICT).

To reduce its energy consumption, a base station can be turned off for some minutes or hours when there are no users to serve [2]. This technique is especially interesting in dense small cell networks [3]. Another solution is to use discontinuous transmission (cell DTx) [4]. With this solution, the base station switches to sleep mode during a few microseconds. During sleep modes, only some of the elements of the RF chain such as the power amplifier are switched-off when there are no signal to transmit (either reference signal or users data). Cell DTx could be very advantageous in the LTE standard, indeed, in this standard, each $10 \mathrm{~ms}$ frame is divided into ten $1 \mathrm{~ms}$ subframes and up to six of those subframes can be used for multicast broadcast single frequency network (MBSFN), during which the base station can be switched to sleep mode.

In [5], the authors evaluate the energy saved with MBSFN subframes, and in [6], it is studied how switching the power amplifier on and off can save energy while maintaining the quality of service and cell coverage. However, in these papers, only DTx is used. Moreover, when cell DTx is used with power control (PC), the sleep mode time is adjusted by adapting the users service times (duration of data transmission) and transmit powers. In this case, there is, for each user, an optimal value for the transmit power and service time which minimizes the base station energy consumption. The expression of this minimum was first derived in [7] but the result wasn't extended to multiple access. In [8], the authors proposed some strategies for reducing the base station energy consumption for packet mode channel access. Furthermore, the problem of minimization of the base station energy consumption in time division multiple access (TDMA) was first raised in [9] with simulation results. In [10], the authors use an interior point method to solve this problem. In these two papers, generic methods are used to solve the problem of energy minimization in TDMA. In [11], the authors show that for base stations with a low power consumption during sleep mode, such as picocells and femtocells, the base station energy consumption is minimized with DTx only. In other words, in this case, it is optimal to serve the users with the maximum RF transmit power during the minimum time. In this article, we show that this result is not true for base stations with a higher power consumption during sleep mode such as macrocells.

In this paper, as in [9], [10], we only consider the downlink communications of a base station. We suppose that each user requires a given quality of service (capacity demand). All users are served during a frame of duration $T$. In each frame, the base station adjusts the transmit power for each user and switches to sleep mode until the end of the frame after having served all users. In this case, the problem of base station energy consumption minimization can be written as a convex constrained optimization problem.

In this paper, we first rewrite the problem of base station energy minimization in TDMA to make it solvable. Then, we use the Karush-Kuhn-Tucker (KKT) conditions to solve it. We show that for microcells and macrocells, it is not always optimal to switch to sleep mode and that in some cases, the energy consumption can be minimized using power control only. When it is optimal to switch to sleep mode, we show that the problem can be solved with closed-form expressions. Furthermore, we propose an efficient algorithm which uses these closed-form expressions to compute the optimal service time and transmit power of each user in TDMA. Finally, we show that, when the use of power control only is optimal (the base station doesn't switch to sleep mode), the problem can be changed into a root-finding problem which can be solved with the Newton's method.

The rest of this paper is organized as follows. The system model is introduced in section II and the optimization problem in section III. The algorithm proposed is described in section IV. In section V, some numerical results are presented and section VI concludes this paper. 


\section{SySTEM MODEL}

In this paper, we suppose that a Base Station (BS) serves $N_{u}$ users. The base station knows the throughput demand (capacity constraint denoted $C_{i}$ ) and the channel gain $G_{i}$ of each user. In each frame of duration $T$, we denote $t_{i}$ the service time of user $i$ which is the time spent to serve him during the frame. We define the proportion of time used to serve user $i$ as:

$$
\mu_{i}=\frac{t_{i}}{T}
$$

We denote $P_{T_{X}}^{i}$ the transmit power used for this user. The total power consumption of a base station (processing, active cooling and transmit power) can be modeled as [12]:

$$
\left\{\begin{array}{cccc}
P_{\text {supply }} & = & P_{0}+m P_{T_{X}} & \text { if } 0<P_{T_{X}} \leq P_{\text {max }} \\
P_{\text {supply }}= & P_{s} & \text { else }
\end{array}\right.
$$

Where $P_{0}$ is the static power consumption, $P_{T_{X}}$ the RF transmit power which is lower than the maximum RF transmit power of the base station denoted $P_{\max } . m$ is the coefficient of the load dependence and $P_{s}$ the power consumption of the base station during sleep mode.

In [12] the authors list some realistic values for $P_{0}, P_{s}$, $P_{\max }$, and $m$. Those values are mentioned in table I.

TABLE I

POWER VALUES FOR DIFFERENT BASE STATIONS TYPES FROM [12]

\begin{tabular}{|c|c|c|c|c|}
\hline BS type & $P_{\max }(\mathrm{W})$ & $P_{0}(\mathrm{~W})$ & $m$ & $P_{s}(\mathrm{~W})$ \\
\hline Macro & 20.0 & 130.0 & 4.7 & 75.0 \\
\hline Micro & 6.3 & 56.0 & 2.6 & 39.0 \\
\hline Pico & 0.13 & 6.8 & 4.0 & 4.3 \\
\hline Femto & 0.05 & 4.8 & 8.0 & 2.9 \\
\hline
\end{tabular}

As illustrated in Fig.1, we suppose that TDMA is used to manage multiple access and that the base station switches to sleep mode until the end of the frame after serving all users. In this case, all users are served over all the available band of bandwidth $B$.

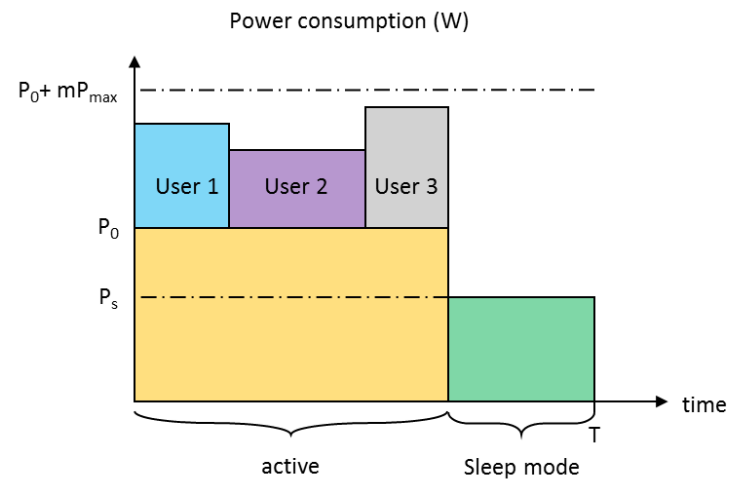

Fig. 1. Variation of the base station power consumption during a frame. The base station switches to sleep mode until the end of the frame after serving all users.

The transmit power and the proportion of time used for each user are computed by the base station to minimize its energy consumption while satisfying the users' capacity demands (QoS). The expression of the capacity demand of user $i$ is:

$$
C_{i}=B \mu_{i} \log _{2}\left(1+\frac{P_{T_{X}}^{i} G_{i}}{B}\right) \quad(b i t s / s)
$$

where $G_{i}=\frac{\left|h_{i}\right|^{2}}{N_{0}}$ is the channel gain (attenuation, antenna gain, thermal noise, noise figure) and the ratio

$$
\rho_{i}=\frac{P_{T_{X}}^{i} G_{i}}{B}
$$

is the Signal-to-Noise Ratio (SNR) of user $i$ which depends on the transmit power used for him.

\section{PRoblem Formulation}

First of all, the energy consumed during a frame is the sum of the energy consumed during sleep mode and when the base station is active. Its expression can be deduced from equation (2):

$$
E=\left(1-\sum_{i=1}^{N_{u}} t_{i}\right) P_{s}+\sum_{i=1}^{N_{u}} t_{i}\left(P_{0}+m P_{T_{X}}^{i}\right)
$$

Then, the expression of the transmit power $P_{T_{X}}^{i}$ required to meet the capacity constraint $C_{i}$ of user $i$ can be deduced from (3):

$$
P_{T_{X}}^{i}=\frac{B}{G_{i}}\left(2^{\frac{C_{i}}{B \mu_{i}}}-1\right) .
$$

Besides, the base station energy consumption can be minimized by minimizing the average power consumption. The average power consumption during a frame is the ratio between the energy consumption during the frame and the frame duration. Its expression can be deduced from (5) and (6):

$$
\begin{gathered}
P_{m}=\underbrace{\left(\sum_{i=1}^{N_{u}} \mu_{i}\right) P_{0}+\left(1-\sum_{i=1}^{N_{u}} \mu_{i}\right) P_{s}}_{\text {Mean static power consumption } P_{m}^{\text {stat }}} \\
+\underbrace{m B \sum_{i=1}^{N_{u}} \mu_{i} \frac{1}{G_{i}}\left(2^{\frac{C_{i}}{B \mu_{i}}}-1\right)}_{\text {Mean dynamic power consumption } P_{m}^{\text {dyn }}} .
\end{gathered}
$$

By analysing separately the two terms of equation (7), we note that the mean static power consumption is minimized by minimizing the users service time, whereas the dynamic power consumption is minimized by minimizing the transmit power, i.e. by maximizing the service time. Thus, minimizing $P_{m}$ amounts to find the best trade-off between these two extreme solutions.

Furthermore, the total service time can't be longer than the frame duration. This condition leads to the following constraint:

$$
\sum_{i=1}^{N_{u}} \mu_{i} \leq 1 .
$$

Moreover, the RF transmit power can't be higher than the maximum RF transmit power denoted $P_{\max }$. To facilitate the resolution of the problem, we change the constraint on the 
maximum transmit power into a constraint on $\mu_{i}$. We denote $\mu_{i \min }$ as the minimum service time for user $i . \mu_{i \text { min }}$ is the time required by the base station to serve user $i$ with the maximum transmit power. $\mu_{i \text { min }}$ is a function of $C_{i}$ and $G_{i}$ and can be derived from equation (3):

$$
\mu_{i \min }=\mu_{\min }\left(C_{i}, G_{i}\right) \quad \forall i \llbracket 1 ; N_{u} \rrbracket
$$

Where the function $\mu_{\min }$ is defined as:

$$
\mu_{\text {min }}(C, G)=\frac{C}{B \log _{2}\left(1+\frac{P_{\max } G}{B}\right)} \quad \forall(C, G) \in \mathbb{R}_{+}^{*} .
$$

If $\mu_{i}<\mu_{i \min }$, then, $P_{T_{X}}^{i}>P_{\max }$ and thus the value of $\mu_{i}$ will be unusable. Therefore, we conclude that it is possible to serve all users if and only if:

$$
\sum_{i=1}^{N_{u}} \mu_{i \min } \leq 1
$$

If equation (11) is not met, the base station can either diminish the QoS of users or refuse to serve some of them.

We finally obtain the new formulation for the problem of minimization of the base station energy consumption

Minimize

$P_{m}=P_{s}+\left(\sum_{i=1}^{N_{u}} \mu_{i}\right)\left(P_{0}-P_{s}\right)+m B \sum_{i=1}^{N_{u}} \frac{\mu_{i}}{G_{i}}\left(2^{\frac{C_{i}}{B \mu_{i}}}-1\right)$

Subject to:

$$
\begin{gathered}
\mu_{i} \geq \mu_{i \min } \quad \forall i \in \llbracket 1 ; N_{u} \rrbracket \\
\sum_{i=1}^{N_{u}} \mu_{i} \leq 1
\end{gathered}
$$

It has been proved in [11] that the function $\left(\mu_{1}, \ldots, \mu_{N_{u}}\right) \mapsto$ $P_{m}$ is convex. Thus, the problem studied is a constrained convex optimization problem. In the rest of this paper, we propose an efficient algorithm to solve this problem.

\section{AlgorithM}

According to the Karush-Kuhn-Tucker (KKT) conditions, in a constrained optimization problem with inequality constraints, each constraint can either be considered as an equality or as a strict inequality (the constraint can be binding or nonbinding). For the problem studied here, a two-step algorithm can efficiently manage the constraints:

1) In a first step, only consider the constraints of (13) (suppose that the constraint of (14) is met). In this case, the base station can compute the optimum service times using closed-form expressions.

2) Then, verify if the constraint of (14) is met. If not, the base station doesn't have to be switched to sleep mode to minimize its energy consumption. In that case, the solution of the optimization problem can't be expressed in a closed-form expression and an iterative algorithm is necessary.

Once the optimal service time for each user was calculated, the transmit power is computed with (6).

\section{A. First step}

The goal of this step is to compute the optimal value of the service time for each user. In other words, we solve here the minimization problem given by equations (12) and (13). To do that, we first compute the value of $\mu_{i}$ which minimizes equation (12). This minimum is denoted $\mu_{i o p t 1}$. Then, we verify if equation (13) is met. If not, $\mu_{i \text { opt } 1}$ is not feasible and the base station energy consumption is minimized by serving the user during $\mu_{i \text { min }}$. We show in this section that the choice between $\mu_{i \text { min }}$ and $\mu_{i o p t 1}$ can be done comparing the users channel gain with a threshold which depends on the characteristics of the base station.

We denote $\mu_{i \text { opt }}$ the optimal service time for the $i^{t h}$ user. $\mu_{i o p t}$ can either be equal to $\mu_{i o p t 1}$ or to $\mu_{i \min } . \mu_{i o p t 1}$ is the value of $\mu_{i}$ which verifies $\frac{\partial P_{m}}{\partial \mu_{i}}=0$. After some mathematical derivations, we derive the expression of $\mu_{i o p t 1}$ :

$$
\mu_{i o p t 1}=\mu_{o p t 1}\left(C_{i}, G_{i}\right) \quad \forall i \in \llbracket 1 ; N_{u} \rrbracket
$$

Where the function $\mu_{o p t 1}(C, G)$ is defined as:

$$
\mu_{\text {opt } 1}(C, G)=\frac{C \ln (2)}{B} \frac{1}{\mathcal{W}\left(e^{-1}\left[\frac{G}{B} \frac{P_{0}-P_{s}}{m}-1\right]\right)+1} .
$$

In this expression, $\mathcal{W}$ denotes the $\mathrm{W}$-Lambert function [13] which is the reciprocal bijection of $x \mapsto x e^{x}$.

According to the KKT conditions, if $\mu_{i o p t 1}$ is lower than $\mu_{i \min }, \mu_{i o p t}$ must be set to $\mu_{i \text { min. }}$. To determine if the constraint of equation (13) is met, we need to know if $\mu_{\text {i opt } 1} \geq \mu_{i \min }$. For that, we define the function:

$$
\rho^{M}(G)=\frac{P_{\max } G}{B} \quad \forall G \in \mathbb{R}_{+}^{*} .
$$

Note that, $\rho_{i}^{M}=\rho^{M}\left(G_{i}\right)$ is the SNR that would have user $i$ if he was served with the maximum transmit power. Moreover, we define the ratio $r$ as:

$$
r=\frac{P_{0}-P_{s}}{m P_{\max }}
$$

For each base station type, the ratio defined in equation (18) allows to assess the power which can be saved by switching to sleep mode compared to the energy which can be saved by reducing the RF transmit power. The larger is $r$, the more interesting it is to switch to sleep mode.

Notice that the function $\mu_{o p t 1}$ can be rewritten as a function of $r, C$ and $\rho^{M}$ and that, similarly, $\mu_{\min }$ can be written as a function of $C$ and $\rho^{M}$. In the following, we denote $\rho_{\text {lim }}^{M}$ the value of $\rho^{M}$ which verifies:

$$
\mu_{\text {opt } 1}\left(C, r, \rho_{\text {lim }}^{M}\right)=\mu_{\min }\left(C, \rho_{\text {lim }}^{M}\right)
$$

Proposition 1. The function $f: r \mapsto \rho_{\text {lim }}^{M}$ is a bijective and strictly increasing function over $\mathbb{R}_{+}^{*}$.

Proof. To prove proposition 1, we first analyse the function $g: \rho_{\text {lim }}^{M} \mapsto r$. For, $\rho_{\text {lim }}^{M} \in \mathbb{R}_{+}^{*}$, we derive from equation (19):

$$
g\left(\rho_{\text {lim }}^{M}\right)=r=\frac{P_{0}-P_{s}}{m P_{\max }}=\left[1+\frac{1}{\rho_{\text {lim }}^{M}}\right] \ln \left(1+\rho_{\text {lim }}^{M}\right)-1 .
$$


The derivative of this expression with respect to $\rho_{\text {lim }}^{M}$ is:

$$
\frac{d g}{d \rho_{l i m}^{M}}=\frac{1}{\rho_{l i m}^{M}}-\frac{1}{\rho_{l i m}^{M 2}} \ln \left(1+\rho_{l i m}^{M}\right)
$$

We now use an inequality of concavity of the logarithm:

$$
\ln (1+x)<x \quad \forall x>0
$$

This inequality proves that the function $g: \rho_{\text {lim }}^{M} \mapsto r$ is a strictly increasing function over $] 0 ;+\infty[$. Thus, this function is bijective and has a reciprocal bijection. This reciprocal bijection, $f: r \mapsto \rho_{\text {lim }}^{M}$ is also bijective and strictly increasing. Which ends the proof.

The consequence of proposition 1 is that for each base station type and for each user, the choice between $\mu_{i \text { min }}$ and $\mu_{\text {i opt } 1}$ can be done by comparing $\rho_{i}^{M}$ and $\rho_{\text {lim }}^{M}$. Indeed, user $i$ must be served during $\mu_{i \text { min }}$ if $\rho_{i}^{M}<\rho_{\text {lim }}^{M}$ and during $\mu_{i \text { opt } 1}$ else. The function $f: r \mapsto \rho_{\text {lim }}^{M}$ and the values of $\rho_{\text {lim }}^{M}$ for the BS types of table I are displayed in Fig. 2.

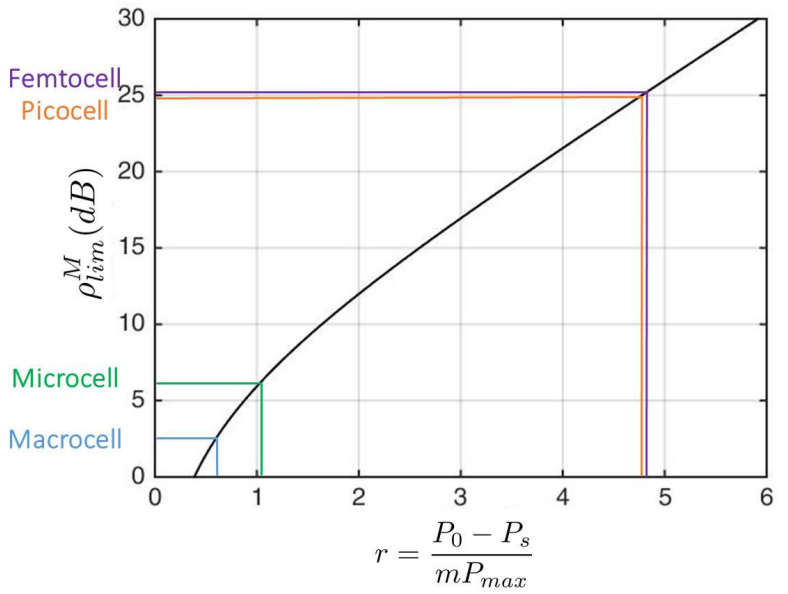

Fig. 2. $\rho_{\text {lim }}^{M}$ versus $r$. This graph can be used to evaluate the value of $\rho_{\text {lim }}^{M}$ for each BS type

Furthermore, if the bandwidth $B$ is also known, the choice between $\mu_{i \text { min }}$ and $\mu_{i \text { opt } 1}$ can be made by comparing the value of $G_{i}$ with a threshold denoted $G_{l i m}$. This threshold can be computed a priori and a base station can compute the optimal service time for each user with the following algorithm:

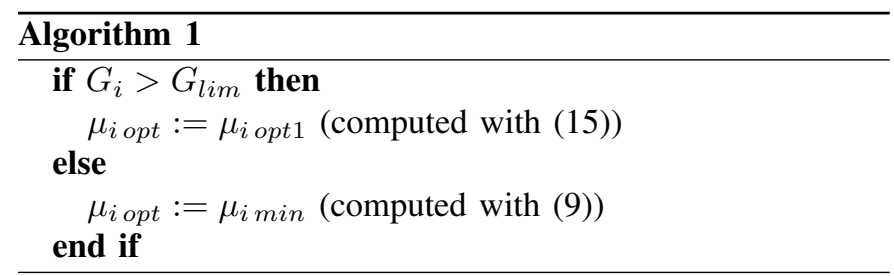

Table II lists the values of $\rho_{\text {lim }}^{M}$ for the base station types introduced in table I and the corresponding values of $G_{l i m}$ for a bandwidth $B=10 \mathrm{MHz}$. We can see in table II, that for macrocells almost all users should be served during $\mu_{i o p t 1}$ whereas for picocells and femtocells almost all users have to be served during $\mu_{i \min }$. It has been shown in [11], that for picocells and femtocells, it is optimal to use DTx only and to serve all users during $\mu_{i m i n}$.

TABLE II

LIMIT VALUES FOR THE CHOICE BETWEEN $\mu_{i}$ min AND $\mu_{i o p t}$

\begin{tabular}{|c|c|c|c|}
\hline BS type & $r$ & $\rho_{\text {lim }}^{M}(d B)$ & $G_{\text {lim }}$ for $B=10 \mathrm{MHz}$ \\
\hline Macro & 0.58 & 2.6 & $9 \times 10^{5}$ \\
\hline Micro & 1.04 & 6.2 & $6.7 \times 10^{6}$ \\
\hline Pico & 4.80 & 25.1 & $2.5 \times 10^{10}$ \\
\hline Femto & 4.75 & 24.9 & $6.1 \times 10^{10}$ \\
\hline
\end{tabular}

This result is not true for macrocells and microcells. Indeed, for these base stations, according to Fig. 2 and table II, the ratio between the dynamic power consumption and the difference $P_{0}-P_{s}$ is lower and most of the users have to be served during a longer time.

\section{B. Second step}

At the end of the first step, we have to verify if the constraint of (14) is met. If it is the case, the users RF transmit power can be computed with (6) and users can be served in an optimal scheme. Else, the value of $\mu_{\text {opt }}$ can't be computed with closedform expressions. The problem of minimization becomes

Minimize

$$
P_{m}=P_{0}+m B \sum_{i=1}^{N_{u}} \frac{\mu_{i}}{G_{i}}\left(2^{\frac{C_{i}}{B \mu_{i}}}-1\right)
$$

Subject to:

$$
\begin{aligned}
& \sum_{i=1}^{N_{u}} \mu_{i}=1 \\
& \mu_{i} \geq \mu_{i \text { min }}
\end{aligned}
$$

In this case, the base station doesn't switch to sleep mode and only power control is used to minimize the base station energy consumption. In this section, we introduce an algorithm for the computation of the power control strategy which minimizes the base station energy consumption. To do that, we first analyse the set of users which have to be served during $\mu_{i \text { min }}$. Then, we use a root-finding method to compute the service time of other users. Note that this algorithm can also be used for a base station without sleep mode. With the new constraint of (24), the expression of the Lagrangian is:

$$
\mathscr{L}=P_{0}+\sum_{i=1}^{N_{u}} \mu_{i} \frac{m B}{G_{i}}\left(2^{\frac{C_{i}}{B \mu_{i}}}-1\right)+K\left(\sum_{i=1}^{N_{u}} \mu_{i}-1\right),
$$

where $K$ is the Lagrange multiplier. The constraints of (25) are not taken into account in this Lagrangian and will be managed independently thereafter.

To solve this problem, we first calculate the expression of the function $K \mapsto \mu_{i}(K)$ which minimizes the Lagrangian (26) and then, the value of $K$ denoted $K_{o p t}$ which verifies (24), i.e. which verifies:

$$
\sum_{i=1}^{N_{\min }} \mu_{i \min }+\sum_{i=N_{\min }+1}^{N_{u}} \mu_{i o p t 2}\left(K_{o p t}\right)-1=0,
$$


where $N_{\min }$ is the number of users served during $\mu_{i \text { min }}$. The expression of $\mu_{\text {iopt } 2}(K)$ which verifies $\frac{\partial \mathscr{L}}{\partial \mu_{i}}\left(\mu_{i \text { opt } 2}\right)=0$ and potentially minimizes the base station energy consumption is:

$$
\begin{aligned}
\mu_{i o p t 2}(K) & =\mu_{o p t 2}\left(K, C_{i}, G_{i}\right) \\
& =\frac{C_{i} \ln (2)}{B} \frac{1}{\mathcal{W}\left(e^{-1}\left[\frac{G_{i}}{B} \frac{K}{m}-1\right]\right)+1} .
\end{aligned}
$$

By comparing (28) and (16), $K_{\text {opt }}$ can be seen as the theoretic value of $P_{0}-P_{s}$ which reduces sufficiently the service time of users to meet the constraint of (27). Thus, $K_{o p t}$ is higher than $P_{0}-P_{s}$ and, because their service time can't be smaller, the users served during $\mu_{i \min }$ at the end of the first step keep the same service time. We denote $N_{m}$ the number of users served during $\mu_{i \text { min }}$ at the end of the first step. To find the set of $N_{\min }$ users which have to be served during $\mu_{i \text { min }}$, we have to analyse the function $K_{m}: G \mapsto K_{m}$ for which:

$$
\mu_{\text {opt } 2}\left(K_{m}, C, G\right)=\mu_{\min }(C, G)
$$

After some mathematical derivations, we derive:

$$
K_{m}(G)=\left[\frac{m B}{G}+m P_{\max }\right] \ln \left(1+\frac{P_{\max } G}{B}\right)-m P_{\max }
$$

Equation (30) allows to compute, for each user, the value $K_{i}=K_{m}\left(G_{i}\right)$ which is the minimum value of $K$ for which user $i$ has to be served during $\mu_{i \min }$. In other words, all the users for which $K_{i}<K_{o p t}$ have to be served during $\mu_{i \text { min }}$ whereas all users for which $K_{i}>K_{\text {opt }}$ have to be served during a longer time to minimize the base station energy consumption. The two following results are used to determine the set of users which have to be served during $\mu_{i \text { min }}$ at the optimum:

1) $K_{m}$ is an increasing function. This can be proved with a proof similar to the one done for proposition 1 .

2) The function $\mu_{o p t 2}$ and consequently the total service time are decreasing functions of $K$.

We can deduce from the first result that, if user $j$ has to be served during $\mu_{j \min }\left(K_{j}<K_{\text {opt }}\right)$, all the users with a lower channel gain must be served during $\mu_{i \text { min }}\left(K_{i}<K_{j}<K_{o p t}\right)$. As a consequence, to find the set of users served during $\mu_{i \text { min }}$, we have to find the user with the higher channel gain served during $\mu_{i \text { min }}\left(N_{\text {min }}^{t h}\right.$ user).

The second result allows to find this user. Indeed, for him, $K_{N_{\min }}<K_{\text {opt }}$ and consequently $\sum_{i=1}^{N_{u}} \mu_{i o p t}\left(K_{N_{\text {min }}}\right)>$ 1. For the user with a channel gain directly above $\sum_{i=1}^{N_{u}} \mu_{\text {i opt }}\left(K_{N_{\min }+1}\right)<1$. As a consequence, to find the set of users served during $\mu_{i \text { min }}$, we can sort the users in ascending order of $G_{i}$. Then, for each user, we compute the value of $K_{i}$ and the corresponding service time until we have a service time lower than 1 . This algorithm is detailed in algorithm 2 .

Once this user has been found, all the users with a lower channel gain must be served during $\mu_{i \text { min }}$, and those with a higher channel gain during $\mu_{\text {iopt } 2}\left(K_{\text {opt }}\right)>\mu_{\text {imin }}$.

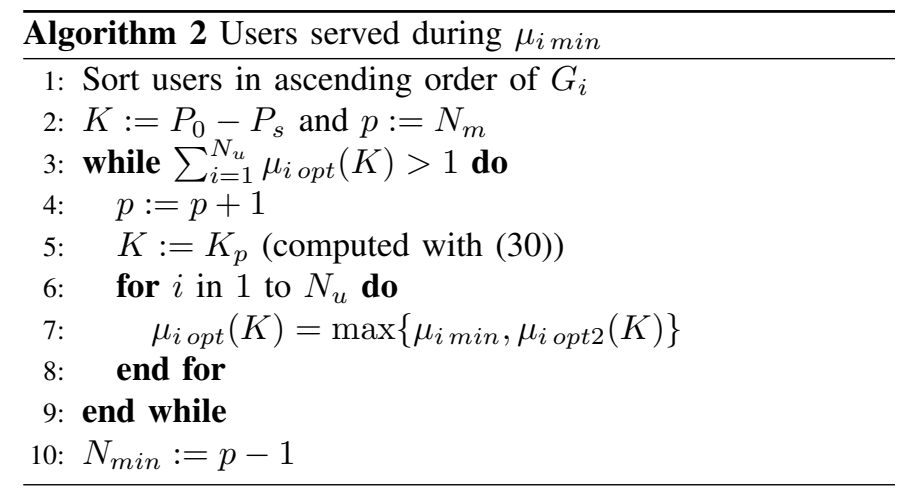

We have determined the set of users which must be served during $\mu_{i \text { min }}$. With the proposed strategy, the search of the optimum is reduced to a one-dimensional problem, and, $K_{\text {opt }}$ can be found with a root-finding algorithm. Moreover, the algorithm presented above also reduces the size of the interval in which lies $K_{o p t}$. Indeed, setting $K_{N_{m}}=P_{0}-P_{s}$, we have:

$$
K_{o p t} \in\left[K_{N_{m i n}} ; K_{N_{m i n}+1}\right]
$$

Finally, root-finding methods can be used to find $K_{o p t}$. Root-finding algorithm are sorted in two categories, bracketing methods (bisection, regula falsi or Brent method) and open domain methods (Newton's method, fixed-point, secant method, etc.) [14]. Bracketing methods are reliable but have a low rate of convergence whereas open domain methods have a high rate of convergence but their convergence is not always guaranteed. Among the cited methods, the Newton's method is the only one which ensures at least a quadratic rate of convergence.

We denote respectively $K_{N_{m i n}}$ and $K_{N_{m i n}+1}$ the bounds of the search interval, $K_{0}$ the starting point of the algorithm and $f$ the function of which we seek the root. The convergence of the Newton's method can be ensured by the following sufficient conditions:

- $f$ has only one root and $f\left(K_{0}\right)>0$

- $f \in \mathcal{C}^{2}\left[K_{N_{\text {min }}} ; K_{N_{\text {min }}+1}\right], f^{\prime}(K) \neq 0$ and $f^{\prime \prime}(K)>0$

- $f\left(K_{N_{m i n}}\right) f\left(K_{N_{m i n}+1}\right)<0$

Proposition 2. If $K_{0}=K_{N_{m i n}}$ the Newton's method applied to the function

$$
f: K \mapsto \sum_{i=1}^{N_{\min }} \mu_{i \min }+\sum_{i=N_{\min }+1}^{N_{u}} \mu_{i o p t 2}(K)-1
$$

converges to its unique root.

Proof. $f \in \mathcal{C}^{2}\left[K_{N_{m i n}} ; K_{N_{m i n}+1}\right]$, and, with the results previously established, $f\left(K_{N_{m i n}+1}\right)<0$ and $f\left(K_{0}\right)=$ $f\left(K_{N_{\text {min }}}\right)>0$. Moreover,

$$
\begin{aligned}
& \mu_{i \text { opt } 2}^{\prime}(K)= \\
& -\frac{e^{-1} C_{i} \ln (2) G_{i}}{B^{2} m} \frac{e^{-\mathcal{W}\left(e^{-1}\left[\frac{G_{i}}{B} \frac{K}{m}-1\right]\right)}}{\left(\mathcal{W}\left(e^{-1}\left[\frac{G_{i}}{B} \frac{K}{m}-1\right]\right)+1\right)^{3}}<0
\end{aligned}
$$

Thus, $f^{\prime} \neq 0$ as it is the sum of strictly negative functions. Therefore, according to the bijection theorem, $f$ has only one root. 
Finally,

$$
\begin{aligned}
\mu_{\text {iopt } 2}^{\prime \prime}(K)= & \frac{e^{-2} C_{i} \ln (2) G_{i}^{2}}{B^{3} m^{2}} \frac{e^{-2 \mathcal{W}\left(e^{-1}\left[\frac{G_{i}}{B} \frac{K}{m}-1\right]\right)}}{\left(\mathcal{W}\left(e^{-1}\left[\frac{G_{i}}{B} \frac{K}{m}-1\right]\right)+1\right)^{5}} \\
& \times\left(\mathcal{W}\left(e^{-1}\left[\frac{G_{i}}{B} \frac{K}{m}-1\right]\right)+4\right)>0
\end{aligned}
$$

Thus $f^{\prime \prime}>0$ as the sum of strictly positive functions. The three sufficient conditions are verified. We have proved that the proposed algorithm converges.

Note that, the value $\tilde{K}$ given by this algorithm is lower than the theoretical value of $K$ and thus $\sum_{i=1}^{N_{u}} \mu_{i \text { opt }}(\tilde{K})>1$. As a consequence, $\tilde{K}$ will be inoperable in practice. This problem can be solved seeking the value of $\tilde{K}$ which verifies:

$$
\sum_{i=1}^{N_{u}} \mu_{i o p t}(\tilde{K})=1-\eta
$$

Where $\eta$ denotes the maximum accepted error. The algorithm should be stopped when $\sum_{i=1}^{N_{u}} \mu_{i \text { opt }}(\tilde{K})<1$.

Once the service time is computed for all users, we use equation (6) to compute the transmit power.

\section{NUMERICAL RESUltS}

In this section, numerical simulations are used to validate our theoretical results. Besides, we evaluate the energy saved by the use of both DTx and power control. Moreover, we analyse the rate of convergence of the second step of the algorithm.

We suppose a macrocell whose characteristics are those of table I. A band of bandwidth $B=10 \mathrm{MHz}$ is used with a central frequency of $2 \mathrm{GHz}$. A Poisson process is used to generate the number of users. The mean number of users in the cell coverage is equal to 10 . The users are uniformly distributed and the distance between users and the base station varies between 300 and $1500 \mathrm{~m}$. The path loss is computed with the Cost 231 Hata model and a log-normal shadowing with a standard deviation of $10 \mathrm{~dB}$ is added. We suppose that the antenna gain of the base station is $10 \mathrm{dBi}$ and the noise figure is set to $9 d B$ for all users. We suppose that all users have the same capacity constraint $C_{i}=C, \forall i \in \llbracket 1 ; N_{u} \rrbracket$.

We first compare the mean power consumption of the base station during a frame with three different strategies:

- Only DTx is used, all users are served during $\mu_{i \min }$ and the transmit power is equal to $P_{\max }$ for all users.

- Power control is used optimally. In this case, the base station never switches to sleep mode and the second part of the algorithm introduced in section IV-B is used to compute the optimum service time for all users.

- The optimal strategy proposed in this paper is used.

The mean power consumptions are compared for different values of the capacity constraint. The results are displayed in Fig.3. When the capacity constraint is low, most of the energy consumed by the base station is static. When the QoS increases, the proportion of dynamic power consumption (part of the power consumption which depends on the RF transmit

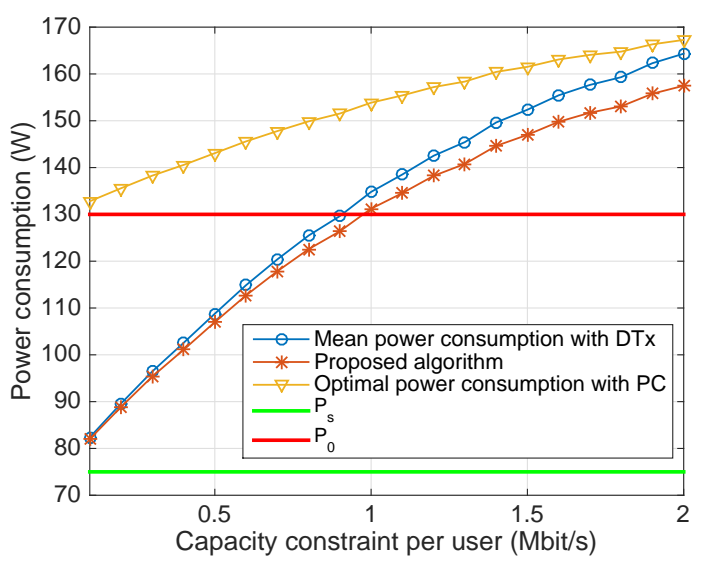

Fig. 3. Comparison between the optimal strategy, the use of power control only and the use of cell DTx only

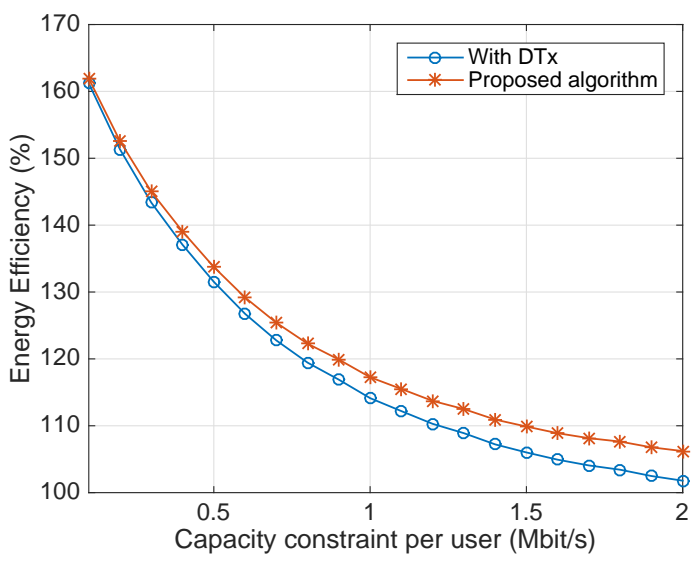

Fig. 4. Comparison of the EE of the three strategies. The EE of the two strategies with DTx are normalized by the EE of the power control strategy

power) increases. As a consequence, the power consumption with the optimal strategy moves away from the power consumption with DTx only (optimal strategy for the static power consumption) and approaches the power consumption with power control (optimal strategy for the dynamic power consumption). The optimal strategy allows to save up to $40 \%$ of the total energy consumption compared to the use of power control and 4\% compared to the use of DTx only.

We now compare the Energy Efficiency (EE) of the three strategies. The EE of the two strategies with DTX (optimal and with DTx only) are divided by the EE of the optimal power control strategy. The results are displayed in Fig.4. For low capacity constraint, the EE of the two DTx strategies is $60 \%$ higher than that with power control only. The gain of the DTx strategies decreases as the capacity constraint increases. This also reflects an increase of the dynamic power consumption.

To assess the evolution of the dynamic power consumption of the base station, we display in Fig.5 the evolution of the proportion of dynamic power consumption (ratio between the dynamic power consumption and the total power consumption). The proportion of dynamic power consumption greatly increases with the capacity constraint. Moreover, with DTx only, the static power consumption is minimum and the dynamic power consumption represents a larger part of the 


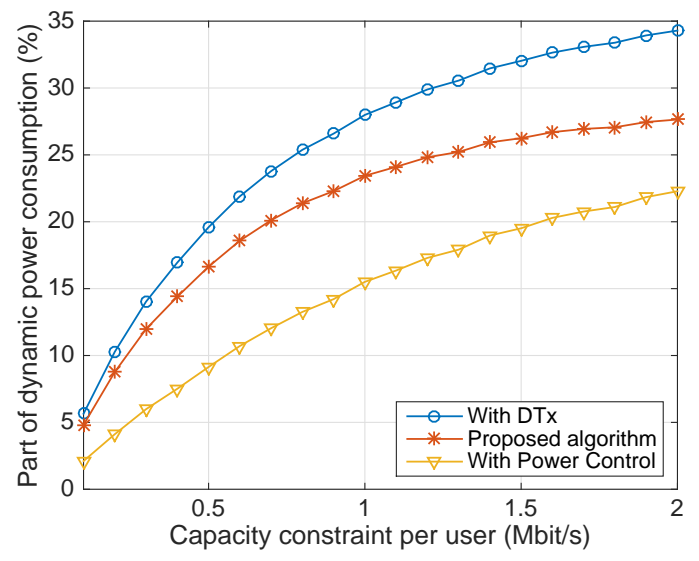

Fig. 5. Proportion of dynamic power consumption

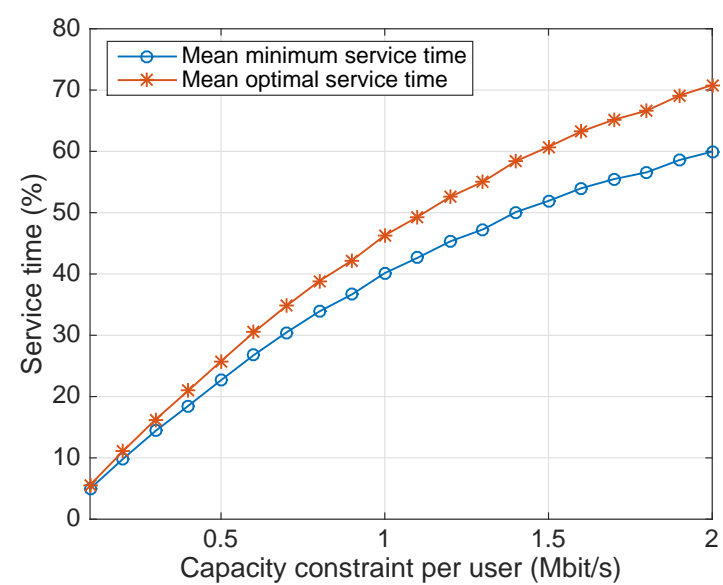

Fig. 6. Comparison between the minimum service time and the optimal service time

base station power consumption (up to $34 \%$ for a capacity constraint of $2 \mathrm{Mbits} / \mathrm{s})$. On the contrary, the power control strategy minimizes the proportion of dynamic power. With power control only, the proportion of dynamic power doesn't exceed $23 \%$.

Finally, we analyse the proportion of time used by the base station to serve all users. The minimum total service time and the optimum total service time are compared for different values of the capacity demand. The results are shown in Fig.6.

On average, the optimal service time is between 12 and $18 \%$ higher than the minimum service time. This percentage changes slowly with the capacity constraint. Indeed, since the capacity constraint is the same for all users, the ratio between the total service times doesn't depend directly on the capacity demand and only varies because the sets of users considered (which verifies $\sum_{i=1}^{N_{u}} \mu_{i \text { min }} \leq 1$ ) change with capacity constraint. When the capacity constraint increases, $\sum_{i=1}^{N_{u}} \mu_{i o p t}$ increases and the constraint of (14) is more often a binding constraint and the second part of the algorithm is used more frequently. The constraint of equation (14) is a binding constraint for $0.1 \%$ of the realizations for a capacity constraint of $0.5 \mathrm{Mbits} / \mathrm{s}, 1.5 \%$ for $1 \mathrm{Mbits} / \mathrm{s}$ and rises to 13.8 $\%$ for a capacity constraint of $2 \mathrm{Mbits} / \mathrm{s}$.

Finally, for simulation, an error of $0.1 \%(\eta=0.001)$ was tolerated and when the second step of the algorithm was necessary, the mean number of iterations was between 2 and 3 . This result highlights the efficiency of the proposed algorithm.

\section{CONCLUSION}

In this paper, we propose a new efficient algorithm for the computation of the optimal service time and transmit power of a base station in TDMA. The proposed optimal strategy significantly reduces the energy consumption of the base station and allows to save up to $40 \%$ of the base station energy consumption compared to the use of power control only and up to $4 \%$ compared to the use of DTx only.

In this paper, we study the optimal use of DTx and power control for a TDMA base station. However, in most of the cellular networks, FDMA is used to manage multiple access and the problem of minimization of the base station energy consumption in FDMA in a multipath fading channel will be addressed in future work.

\section{ACKNOWLEDGMENT}

Part of this work is supported by the project SOGREEN, which is funded by the French national research agency, under the grant agreement coded: N ANR-14-CE28-0025-02 and by Région Bretagne, France.

\section{REFERENCES}

[1] A. Fehske, G. Fettweis, J. Malmodin, and G. Biczok, "The global footprint of mobile communications: The ecological and economic perspective," Communications Magazine, IEEE, vol. 49, pp. 55-62, August 2011.

[2] L. Saker, S.-E. Elayoubi, and T. Chahed, "Minimizing energy consumption via sleep mode in green base station," in Wireless Communications and Networking Conference (WCNC), 2010 IEEE, pp. 1-6, April 2010.

[3] I. Ashraf, F. Boccardi, and L. Ho, "Sleep mode techniques for small cell deployments," IEEE Communications Magazine, vol. 49, pp. 7279, August 2011.

[4] T. Chen and al., "Network energy saving technologies for green wireless access networks," IEEE Wireless Communications, vol. 18, pp. 30-38, October 2011.

[5] P. Frenger and al., "Reducing energy consumption in lte with cell dtx," in Vehicular Technology Conference (VTC Spring), 2011 IEEE 73rd, pp. 1-5, May 2011.

[6] A. Chatzipapas, S. Alouf, and V. Mancuso, "On the minimization of power consumption in base stations using on/off power amplifiers," in Online Conference on Green Communications (GreenCom), 2011 IEEE, pp. 18-23, Sept 2011.

[7] R. Wang, J. Thompson, H. Haas, and P. Grant, "Sleep mode design for green base stations," Communications, IET, vol. 5, pp. 2606-2616, Dec 2011.

[8] J. Wu, S. Zhou, and Z. Niu, "Traffic-aware base station sleeping control and power matching for energy-delay tradeoffs in green cellular networks," Wireless Communications, IEEE Transactions on, vol. 12, pp. 4196-4209, August 2013.

[9] H. Holtkamp, G. Auer, and H. Haas, "On minimizing base station power consumption," in Vehicular Technology Conference (VTC Fall), 2011 IEEE, pp. 1-5, Sept 2011.

[10] H. Holtkamp, G. Auer, S. Bazzi, and H. Haas, "Minimizing base station power consumption," IEEE Journal on Selected Areas in Communications, vol. 32, pp. 297-306, February 2014.

[11] R. Bonnefoi, C. Moy, and J. Palicot, "Dynamic sleep mode for minimizing a femtocell power consumption," in CROWNCOM, May 2016.

[12] G. Auer and al., "How much energy is needed to run a wireless network?," Wireless Communications, IEEE, vol. 18, pp. 40-49, October 2011.

[13] R. Corless and al., "On the lambertw function," Advances in Computational Mathematics, vol. 5, no. 1, pp. 329-359, 1996.

[14] J. D. Hoffman, Numerical methods for engineers and scientists. New York, NY: McGraw-Hill, 1992. 